\title{
Achieving continuous improvement in laboratory organization through performance measurements: a seven-year experience
}

\author{
Maria Salinas ${ }^{1}$, Maite López-Garrigós ${ }^{1, *}$, Mercedes \\ Gutiérrez $^{1}$, Javier Lugo ${ }^{1}$, Jose Vicente Sirvent ${ }^{1}$ and \\ Joaquin Uris ${ }^{2}$ \\ ${ }^{1}$ Department of Clinical Laboratory, University Hospital of \\ San Juan, Alicante, Spain \\ ${ }^{2}$ Department of Public Health, University of Alicante, \\ Alicante, Spain
}

\begin{abstract}
Background: Laboratory performance can be measured using a set of model key performance indicators (KPIs). The design and implementation of KPIs are important issues. KPI results from 7 years are reported and their implementation, monitoring, objectives, interventions, result reporting and delivery are analyzed.

Methods: The KPIs of the entire laboratory process were obtained using Laboratory Information System (LIS) registers. These were collected automatically using a data warehouse application, spreadsheets and external quality program reports. Customer satisfaction was assessed using surveys. Nine model laboratory KPIs were proposed and measured.

Results: The results of some examples of KPIs used in our laboratory are reported. Their corrective measurements or the implementation of objectives led to improvement in the associated KPIs results.

Conclusions: Measurement of laboratory performance using KPIs and a data warehouse application that continuously collects registers and calculates KPIs confirmed the reliability of indicators, indicator acceptability and usability for users, and continuous process improvement.

Clin Chem Lab Med 2010;48:57-61.
\end{abstract}

Keywords: laboratories; quality assessment; quality indicators; quality of health care; total quality management.

\section{Introduction}

The rising cost of health care has stimulated interest in developing methods to increase the efficiency of health-care

*Corresponding author: Maite López-Garrigós, Servicio de Laboratorio Clínico, Hospital Universitario de San Juan, Carretera Alicante-Valencia, s/n, 03550, Alicante, Spain

Phone: +34-96-5938877, Fax: +34-96-5938683,

E-mail: lopez_marter@gva.es

Received August 19, 2009; accepted September 14, 2009;

previously published online November 18, 2009 organizations through adoption of technological advances (1). However, it is paradoxical that clinical laboratories have embraced most technological advances for improving analytical processes, but have been slow to measure laboratory performance using key performance indicators (KPIs).

A KPI is an objective measure for evaluating critical health care domains (patient safety, effectiveness, equity, patient-centeredness, timeliness and efficiency) (2). Assessment of the quality of laboratory services using KPIs requires a systematic, transparent, and consistent approach to collection and analysis of data.

The goal of the present study was to show how laboratory performance is measured using a set of defined KPIs. The results obtained over 7 years using these indicators are reported, and the design, implementation, monitoring, objectives, interventions, result reporting and delivery in daily practice are described.

\section{Materials and methods}

The laboratory was an ISO 9001:2008-certified laboratory affiliated with a public university hospital serving a population of 234,403. It provides care for inpatients and outpatients in primary care centers. Samples were collected by a courier, transported to the laboratory sample reception desk for classification and distributed to the appropriate units where testing was conducted. Once the laboratory physician verified the test results, clinicians could automatically consult the report via the intranet. The clinical laboratory includes chemistry and hematology testing. In 2008, 262,100 requests and 2,668,984 tests were preformed.

The registers used to build the nine model KPIs were "Intern Laboratory Information System (LIS) data" (test registration and test validation time and date), "Daily LIS data" (data necessary for daily work: demographic information, tests requested, test results, coded test results, etc.), and "LIS quality tests" (data entered manually that is not necessary for daily work but records information on incidents that occur during the course of the request). The registers and KPIs were collected and calculated automatically by the LIS using a software program based on OLAP cubes (Omnium by Roche Diagnostics ${ }^{\circledR}$ SUNSET Technologies, Girona, Spain). Transport and analytical KPI registers were recorded on spreadsheets and in External Quality Program reports, respectively. Customer satisfaction was assessed using internal surveys. Surveys used a scale from 0 to $10(0$, poor and 10 , excellent).

The KPI design is shown in Table 1. KPIs were classified as prepre-analytical, pre-analytical, analytical and post-analytical according to the laboratory process that they measured. The indicators were selected to monitor all stages of the entire test process. 
The pre-pre-analytical KPI, or appropriateness indicator, referred to the test request. It measured the number of tests processed per control tests requested.

Patient satisfaction surveys with respect to phlebotomy were considered as one of the four pre-analytical indicators. The KPI demographic register revealed any omission of demographic items (age, requesting doctor, suspected diagnosis, etc.) per total requests. The next KPI referred to unsuitable specimens. These indicators were monitored by defining pre-analytical sample error as a rejected specimen: any sample that was not suitable for one or more tests in the total order because the specimen did not meet acceptability criteria, or the sample was not received (3). The pre-analytical error (coded result that informed of a specific error and recommended collection of a new specimen) and the sample in which the error occurred were collected to calculate the KPI. The last pre-analytical KPI was the transport indicator. The data used to calculate the transport KPI was the number of days that the courier failed to meet the established time objective.

The analytical KPIs were calculated from the external quality program report. This report was based on Six-Sigma results (4) and reported every 3 months to each functional unit.

The post-analytical KPIs showed the percentage of key tests requested that were completed, verified and made available to the requesting doctor on the day of phlebotomy, and manual test turnaround time (TAT) in days (TAT between test registration and verification). Clinician satisfaction with laboratory service was also considered in the post-analytical KPIs.

All the KPIs were expressed as percentages, except for TAT (days) and customer satisfaction KPIs (average score). One KPI result for every model KPI from January 2002 to February 2009 is shown. Indicator results were entered into the SPSS 14.0 for Windows (SPSS Inc, Chicago, IL, USA) and Microsoft Office Excel 2003 for statistical analysis.

\section{Results}

Six KPI results are shown in Figure 1, arranged from A to $\mathrm{F}$ and classified according to the laboratory process. Indi- cator results not shown in the Figure are described in the text.

The pre-pre-analytical KPI (test appropriateness indicator) was the ratio of requests for $\mathrm{C}$-reactive protein (CRP) to requests for glucose by the emergency department (ED). Given the increase in ED stat CRP requests (9285, 16,997 and 20,753 in 2006, 2007 and 2008, respectively), a multidisciplinary working group consisting of emergency room, laboratory, rheumatology, pediatric and infectious disease clinicians was established to design a CRP request protocol for implementation in clinical sessions. The decline in CRP requests lasted 5 months only. Subsequently, the protocol was circulated every 2 months with a short note indicating the importance of an appropriate request for CRP, and KPI evolution to every clinician, but without success (Figure 1A).

Patient requests containing the correct demographic data (age) improved when automatic collection of demographic registers from a government database was implemented in April 2008 (Figure 1B).

Two corrective measures were introduced into transport circuits (May 2005 and September 2007), which rapidly reduced the number of specific transport KPIs (Figure 1C). These interventions consisted of reorganizing the sample collection route among the decentralized phlebotomy centers.

As an example of an unsuitable specimen KPI, we reported the number of samples clotted and unavailable for measurement of the erythrocyte sedimentation rate (ESR). The removal of a specific ESR tube in July 2003 resulted in an immediate improvement of the number of clotted and unavailable ESR sample KPIs (Figure 1D). As a pre-analytical indicator, we used patient satisfaction with phlebotomy, which improved over the past several years (average scores 7.5, 7.7, 7.9, 8 and 8.3 since 2004).

The analytical indicator report showed the number of tests with Sigma value $<3$ and with values $>3$. The Sigma $<3$ bar indicated the number of tests that did not meet the target. This report was sent monthly to all functional units in the

Table 1 Nine model key performance indicators.

\begin{tabular}{|c|c|c|c|}
\hline Indicator & Source register & Design & Periodicity \\
\hline \multicolumn{4}{|l|}{ Pre-pre-analytical } \\
\hline Appropriateness test request & Daily LIS data & Tests requested/control tests requested & Monthly \\
\hline \multicolumn{4}{|l|}{ Pre-analytical } \\
\hline Customer satisfaction & Survey & Average score & Annually \\
\hline Demographic register & Daily LIS data & Incorrect demographic register requests/total requests & Monthly \\
\hline Unsuitable specimen & Daily LIS data & Unsuitable specimen/total specimens & Monthly \\
\hline Transport & Spreadsheet & $\begin{array}{l}\text { Days that the courier does not meet established time } \\
\text { objective/total days }\end{array}$ & Monthly \\
\hline \multicolumn{4}{|l|}{ Analytical } \\
\hline Proficiency testing & $\begin{array}{l}\text { External quality } \\
\text { program }\end{array}$ & Test with Sigma value $>3 /$ total tests & Quarterly \\
\hline \multicolumn{4}{|l|}{ Post-analytical } \\
\hline Turnaround time & Intern LIS data & Median (TAT, between test register and verification) & Monthly \\
\hline Verified in the phlebotomy day & Intern LIS data & Key tests verified the phlebotomy day/total key tests & Monthly \\
\hline Clinician satisfaction & Survey & Average score & Annually \\
\hline
\end{tabular}

Nine key performance indicators, including the name, construction, and periodicity of delivery of indicator results. 


\section{A}

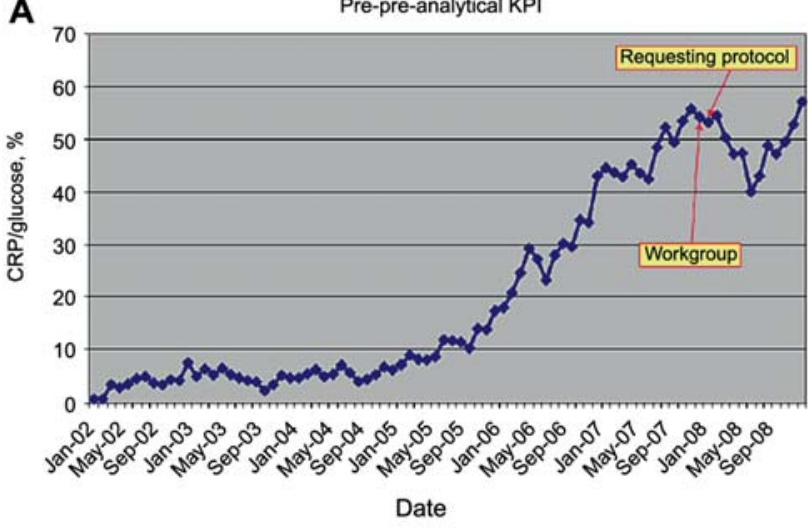

C

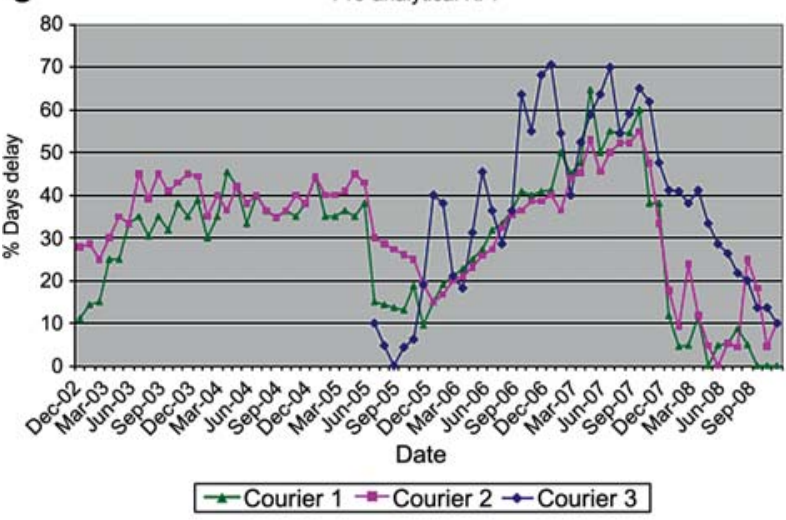

B
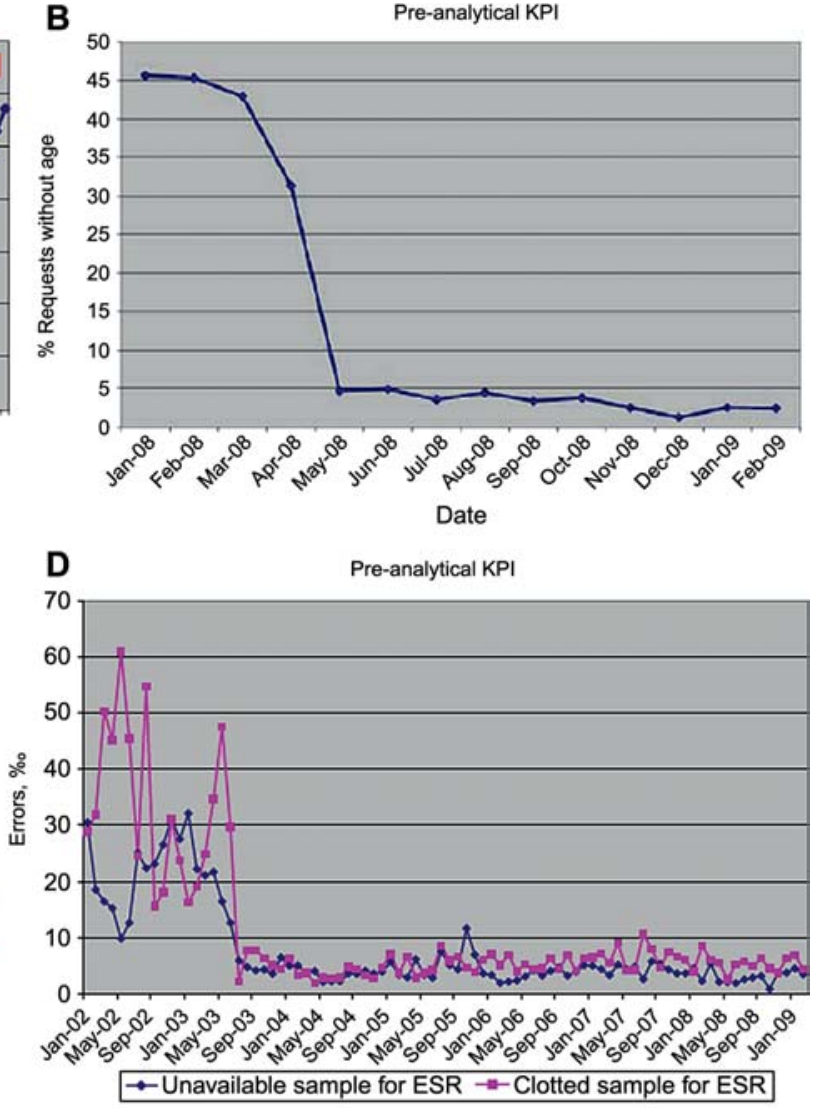

E

$F$

Post-analytical KPIs
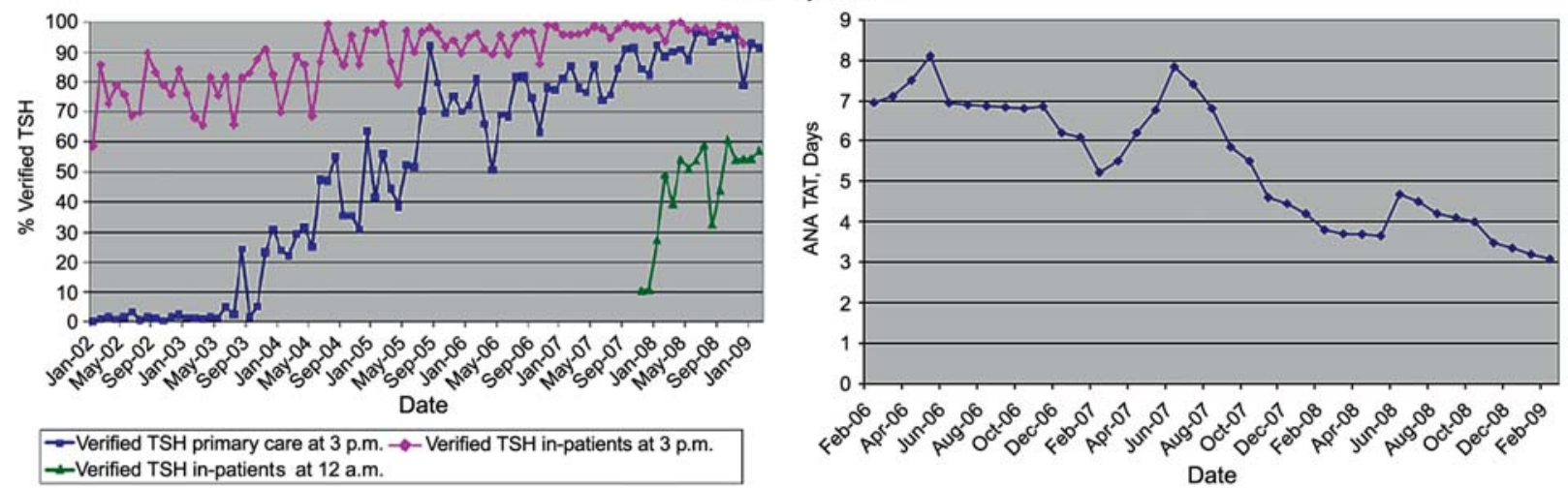

Figure 1. Results of key performance indicators.

The results of six KPIs representative of steps in the laboratory process. (A) Appropriateness test request indicator: measurement of Creactive protein (CRP) per glucose request in the ED. (B) Demographic register indicator: \% of requests without age per total requests. (C) Transport indicator: the number of days that the courier does meet target delivery time per total number of days. (D) Unsuitable specimen indicator: the number of clotted samples and samples unavailable for ESR, per thousand ESR samples collected. (E) Verified on the day of phlebotomy indicator: the monthly delivery of TSH verified at 3 p.m., and subsequent implementation of the objectives ( $99 \%$ for inpatients and $90 \%$ for primary care patients since May 2006) and recently (November 2008). Eighty-five percent for inpatients at 12 a.m. on the day of phlebotomy indicated progressive improvement in the indicator result. (F) Turnaround time indicator: median TAT for ANA.

laboratory. For example, in January 2009 in the chemistry functional unit, all the tests were Sigma $>3$ except for albumin, calcium, sodium and potassium.

Thyroid-stimulating hormone (TSH), verified on the day of phlebotomy, showed progressive improvement in the indi- cator result (Figure 1E). The TAT for antinuclear antibodies (ANA) for samples from inpatients also improved after objective implementation (3 days) in June 2007 (Figure 1F).

Clinician satisfaction (post-analytical indicator) improved over the past several years from 7 to 8 since 2004 . 


\section{Discussion}

The results of a series of KPIs that are representative of every step of the laboratory process are reported. As Shahangian states, there is a general lack of evidence to support the importance, scientific soundness and usefulness of most of these indicators because laboratories generally do not publish their internal monitoring data (5). Therefore, our aim was to elucidate aspects of the indicators for the purpose of continuous quality improvement. However, each laboratory should design its own indicators based on laboratory organization and processes (6).

The pre-pre-analytical KPI chosen was a simple indicator of test appropriateness. It showed the evolution of a specific test compared with another widely used test. This allows corrective measures to be taken when any monthly deviation occurs, in addition to monitoring the measures. The CRP post-intervention appropriateness indicator rose again, confirming that the intervention established was not as effective as other interventions that were proposed (7). Strategies for managing test appropriateness require ongoing re-education of clinicians with respect to test requests. However, problems arose when we tried to reduce the $24 \mathrm{~h}$ demand for test requests due to high turnover of clinicians and limited laboratory staff.

With regard to pre-analytical KPIs, most of the studies associated with patient identification error indicators referred to inpatient identification wristbands (8), which were not available for outpatients. We proposed using incorrect demographic data register KPIs to detect data needed to verify tests, or contacting the requesting doctor if necessary. The transport indicator was an important key point because early sample delivery favors timely reporting. The courier recorded the delivery time. Awareness that the delivery time is part of an indicator result that is summarized in a monthly report involved the courier in the overall test process and encouraged improved courier performance. The unsuitable sample model KPI was valuable. It can be used for any error and sample, provided that the result of the affected test is coded. Increased patient satisfaction appears to result from improvements in the overall laboratory process.

We included a unique synthetic analytical quality indicator for every functional unit. The advantage was that any KPI deviation was analyzed to establish corrective measures. By increasing the target Sigma value, continuous quality improvement in the functional unit can be achieved.

Post-analytical KPIs were feasible because they were calculated using intern LIS registers. The "TSH verified on phlebotomy day" KPI results rose spectacularly, according to goal implementation. Setting an objective and then monitoring over time made it possible to achieve the objective. It is completely different to state that "we processed all the samples every day" from stating "last month 95\% of TSH requests were verified and made available to the requesting doctor on the day of phlebotomy." ANA TAT results also improved according to the goal established.

Every KPI was designed and later validated in daily practice. Once the KPI was designed and validated, a set of objectives was established. KPIs were monitored and KPI results delivered each month to the personnel involved in the process (laboratory functional unit, clinicians, couriers, etc.). This was done to promote feedback and continuous improvement in processing and laboratory performance, as assessed in the study. This interchange of indicator results promotes "healthy competition", thereby changing corporate culture. Each worker is motivated to improve their personal results and those of the initial stages, which improves the entire process and ultimately benefits patients.

\section{Conclusions}

We demonstrate how laboratory performance could be enhanced using a model design based on a Data Warehouse application that continuously collects registers (using the data required for the daily testing process) and calculates KPIs, which requires indicators that are reliable and acceptable and usable.

The evolution of most of the indicator results over the past years suggests that it is not enough to design and implement indicators. It is essential to set goals and deliver the results to those involved in the process. Continuous improvement in performance can be achieved through communication and teamwork $(9,10)$, which are the basis for patient safety.

\section{Acknowledgements}

We thank Rosa Lillo-Bernabeu for her helpful support and Miguel Angel Lorente-Lopez (Roche Diagnostics, Valencia, Spain) for his excellent technical assistance. We also thank all of the staff of the laboratory for their work.

\section{Conflict of interest statement}

Authors' conflict of interest disclosure: The authors stated that there are no conflicts of interest regarding the publication of this article. The technical assistance by Miguel Angel Lorente-Lopez (Roche Diagnostics) played no role in the design of the study; in the collection, analysis, and interpretation of the data; in the writing of the manuscript; or in the decision to submit the manuscript for publication.

Research funding: None declared.

Employment or leadership: None declared.

Honorarium: None declared.

\section{References}

1. Bossuyt X, Verweire K, Blanckaert N. Laboratory medicine: challenges and opportunities. Clin Chem 2007;53:1730-3.

2. Institute of Medicine Committee on Quality of Health Care in America. To Err is Human: Building a Safer Health System. Washington, DC: National Academies Press, 2000.

3. Jones BA, Calam RR, Howanitz PJ. Chemistry specimen acceptability: a College of American Pathologists Q-Probes study of 453 laboratories. Arch Pathol Lab Med 1997;121:19-26.

4. Westgard JO, Westgard SA. The quality of laboratory testingto- 
day: an assessment of sigma metrics for analytic quality using performance data from proficiency testing surveys and the CLIA criteria for acceptable performance. Am J Clin Pathol 2006;125:343-54.

5. Shahangian S, Snyder SR. Laboratory medicine quality indicators: a review of the literature. Am J Clin Pathol 2009;131: 418-31.

6. Plebani M, Ceriotti F, Messeri G, Ottomano C, Pansini N, Bonini P. Laboratory network of excellence: enhancing patient safety and service effectiveness. Clin Chem Lab Med 2006;44: $150-60$

7. Salinas La Casta M, Flores Pardo E, Lugo Arocena J, Uris
Selles J. Declining demand for laboratory, following the amendment of the application form. Med Clin (Barc) 2008;131: 716.

8. Howanitz PJ, Renner SW, Walsh MK. Continuous wristband monitoring over 2 years decreases identification errors: a College of American Pathologists Q-Tracks Study. Arch Pathol Lab Med 2002;126:809-15.

9. Howanitz PJ. Errors in laboratory medicine: practical lessons to improve patient safety. Arch Pathol Lab Med 2005;129: 1252-61.

10. Boone DJ. How can we make laboratory testing safer? Clin Chem Lab Med 2007;45:708-11. 